\title{
Application of mutant particle swarm optimization for MPPT in photovoltaic system
}

Thi Thom Hoang, Thi Huong Le

Department of Electrical \& Electronic Engineering, Nha Trang University, Vietnam

\begin{tabular}{l}
\hline \hline Article Info \\
\hline Article history: \\
Received Dec 1, 2019 \\
Revised Mar 3, 2020 \\
Accepted Mar 24, 2020 \\
\hline
\end{tabular}

\section{Keywords:}

Incremental conductance particle swarm optimization Maximum power point tracking Photovoltaic system

\begin{abstract}
The $\mathrm{P}-\mathrm{V}$ characteristic of a photovoltaic system (PVs) is non-linear and de-pends entirely on the extreme environmental condition, thus a large amount PV energy is lost in the environment. To enhance the operating efficiency of the PVs, a maximum power point tracking (MPPT) controller is normally equipped in the system. This paper proposes a new mutant particle swarm optimization (MPSO) algorithm for tracking the maximum power point (MPP) in the PVs. The MPSO-based MPPT algorithm not only surmounts the steady-state oscillation (SSO) around the MPP, but also tracks accurately the optimum power under different varying environmental conditions. To demonstrate the effectiveness of the proposed method, MATLAB simulations are implemented in three challenging scenarios to the PV system, including changing irradiation, load variation and partial shading condition (PSC). Furthermore, the obtained results are compared to some of the con-ventional MPPT algorithms, such as incremental conductance (INC) and clas-sical particle swarm optimization (PSO) in order to show the superiority of the proposed approach in improving the efficiency of PVs.
\end{abstract}

Copyright (C) 2020 Institute of Advanced Engineering and Science. All rights reserved.

\section{Corresponding Author:}

Thi Thom Hoang,

Department of Electrical \& Electronic Engineering,

Nha Trang University,

No. 2, Nguyen Dinh Chieu St., Vinh Nguyen, Nha Trang, Vietnam.

Email: thomht@ntu.edu.vn

\section{INTRODUCTION}

The PVs is one of the most popular renewable energy source because of low cost, free maintenance and good for environment [1]. To enhance the utilizing efficiency of PVs, a MPPT controller is normally integrated with a power converter in order to obtain the maximum power generated by the PVs. The P-V characteristic; however, is non-linear because of the complicate environmental conditions, so it is a great challenge for the MPP tracking algorithms.

Up to now, a variety of MPPT methods have been proposed to improve the operating efficiency of the PVs. The most common algorithms are the perturb and observe (P\&O) and the incremental conductance (INC). The $(\mathrm{P} \& \mathrm{O})$ method is on basic of the perturbed voltage obtained from a comparison between the present and previous operating power [2-4]. In spite of simple implement, the drawback of this method is the oscillation around the balance state, resulting in the power losses and the increase of the convergence time. Meanwhile, the (INC) method carries out comparing between the ratio of derivative of conductance and the instantaneous con-ductance in order to remove the loss of tracking direction [5-7]. Nevertheless, the steadystate oscillation ussually appears around MPP because of the dynamic characteristic and the size of perturbation step, decreasing the operating efficiency of PVs [8]. In paper [9], the authors applied the fuzzy logic control (FLC) for MPPT by using three steps: fuzzification, fuzzy rule base table and defuzzification. The disadvantage of this method is the tracking accuracy depending on the number of the member functions. 
To overcome this problem, the artificial neural network (ANN) is used; however, it takes a long time to track the MPP because the PV characteristic varies to its life [10].

For tracking the MPP, the hotspot problem should be concerned in case of PVs under PSC because it can damage PV cells. To overcome this situation, some of adaptive behavious algorithms, including genetic algorithm (GA) [11], differential evolution (DE) [12], and particle swarm optimization (PSO) [13, 14] have been proposed for tracking the global point from PVs. Although this method is quite effective, it still suffers from getting trapped into the local optima and from the exces-sive time requirement.

In this paper, a novel MPSO algorithm has been investigated by removing the worst particle by a mutant particle generated randomly using personal best com-ponents of classical PSO in order to optimize the output power of PVs. The proposed MPSO-based MPPT has a similar structure to the classical PSO and hence it is also capability of avoiding the steady-state oscillation and tracking the MPP under the complicate environmental conditions. Furthermore, the algorithm has faster tracking speed, simpler deployment using a lower cost controller as compared to other conventional MPPT methods. The superiority of the proposed method is capabity of tracking the global peak among multi-local peaks in the PV characteristic provided.

\section{PROBLEM DESCRIPTION}

\subsection{Modelling of the PV module and array}

There are many equivalent circuit models proposed to describe the PV modules; however, the single diode model, as depicted in Figure 1, is the most popular [15].

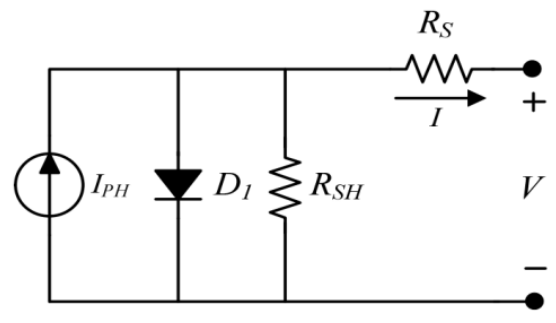

Figure 1. Modelling of a PV module

It can be clear from Figure 1 that the relationship between the output current I and the photocurrent IPH is given as:

$$
I=I_{P H}-I_{D 1}-\left(\frac{V+I R_{S}}{R_{S H}}\right)
$$

where:

$$
I_{D 1}=I_{R S}\left[\exp \left(\frac{V+I R_{S}}{\alpha V_{T}}\right)-1\right]
$$

where IRS is the reverse saturation current of diode D1, $\alpha$ is the diode ideality constant, and VT is the thermal voltage of PV modules that iss expressed by:

$$
V_{T}=k \frac{T}{q}
$$

where $\mathrm{k}$ is the Boltzmann constant, $\mathrm{T}$ is the temperature of the $\mathrm{p}-\mathrm{n}$ junction in Kelvin and $\mathrm{q}$ is the electron charge.

A PV array is composed of many modules, which are connected in a series-parallel matrix (NS x NP), as shown in Figure 2. Mathematically, a PV array can be described as follows: 
$I=N_{P}\left[I_{P H}-I_{R S}\left(I_{P}+2\right)\right]-\left(\left(\frac{V+I R_{S} \lambda}{R_{R S} \lambda}\right)\right)$

where: $I_{p}=\exp \left(\frac{V+I R_{S} \lambda}{V_{T} N_{S}}\right)+\exp \left(\frac{V+I R_{S} \lambda}{(p-1) V_{T} N_{S}}\right)$

$\lambda=\frac{N_{S}}{N_{P}}$

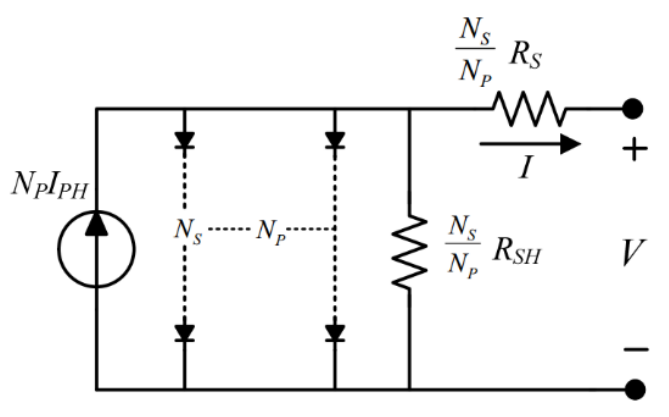

Figure 2. Modelling of a PV array

It can be seen from (4) and (5) that I-V characteristic of the PVs is non-linear, in which the output current varies as a function of the irradiance and temperature, thus decreasing the operating efficiency of PVs.

\subsection{PV arrays under PSC}

When the PVs is set under a uniform solar insolation, a single MPP is obtained on the P-V characteristic curve of the PVs. However, at all time, each cell can receive a different amount of solar energy because some modules might be covered by nearby tree, chimney, or cloud, known as PSC [16, 17]. In this case, the sunlight energy received by the shaded cells is lower than that obtained by the non-shaded cells, leading to the hotspot problem. In other words, the hotpot phenomenon is occurred by absorbing the electric power generated by the non-shaded PV cells, giving rise to damage PV systems [18]. In case of PSC; therefore, each PV cell is normally connected in parallel with a bypass diode to produce other path for the current in order to remove the hotspot problem. However, the disadvantage of inserting a diode is the presence of multiple peaks on the $\mathrm{P}-\mathrm{V}$ characteristics, as shown Figure 3, in which there is only one global optimal point (GP), giving the true MPP $[19,20]$.

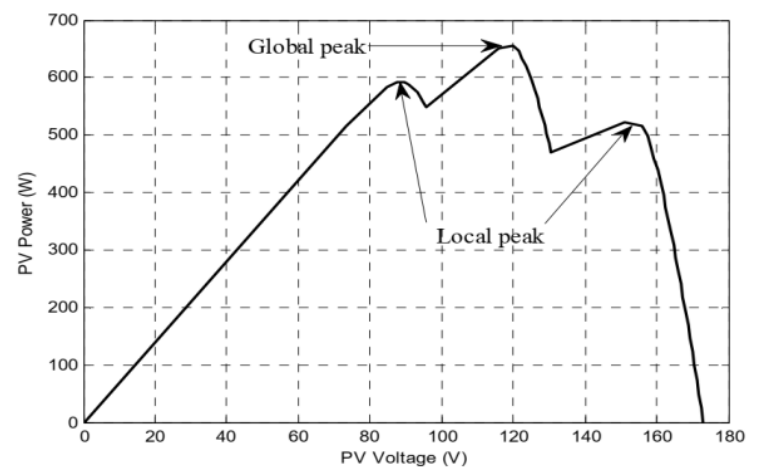

Figure 3. The P-V characteristic during PSC 
To track this GP during PSC, some of the conventional MPPT algorithms are proposed, but they are not effective because of high power loss. To overcome this problem; in this paper, an improved PSO is mentioned for finding the true MPP.

\section{THE MPSO-MPPT ALGORITHM}

PSO is a swarm intelligence, which is introduced in the first time by Kennedy and Eberhart in 1995 [21]. It is inspired by social and cooperative behavior of bird flocking or fish schooling. At first, each particle is initialized by a random solutions and then keeps searching for the optimum solution by updating generations. It can be noted that these particles fly in the search space by following the personal experience (Pbest) and the overall experience (Gbest). More details about the basic conceptualization of PSO can be found in [22-24]. The main disadvantage of PSO is to be sufferred from getting trapped into local minima. In this section, a new version of PSO, namely MPSO is introduced in order to obtain a higher quality solution in the search space. Then, the proposed method is used for finding the true MPP among the multiple peaks on $\mathrm{P}-\mathrm{V}$ characteristic of the tested PVs.

\subsection{MPSO}

In MPSO, the worst particle is replaced by the mutant- particle generated randomly by se-lecting one or more Pbest components of some or all particles of the classical PSO. The mutant-particle is a vector

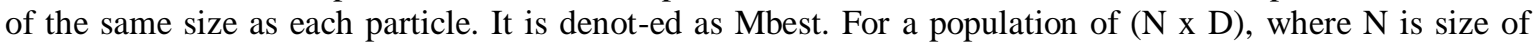
swarm and $\mathrm{D}$ is the dimension of each particle, Mbest can be generated as follows:

$$
\begin{aligned}
& \text { for } \mathrm{q}=1: \mathrm{D} \\
& \text { Mbest } q=\operatorname{Pbest}(\operatorname{randi}(N, 1), q) \\
& \text { End }
\end{aligned}
$$

where randi(N,1) is a function uniformly generating an integer between 0 and $\mathrm{N}$. Thus, in the above for-loop, a total of D integers are generated and the corresponding component from each column is selected from matrix Pbest to form a vector Mbest. This process can be understood more clearly through an example.

Suppose the size of population is 6 and the dimension of each particle is 5. Now, in the for-loop,

\begin{tabular}{|c|c|c|c|c|c|}
\hline \multirow[t]{6}{*}{ Pbest $=$} & Pbest $_{1,1}$ & Pbest $_{1,2}$ & $\underline{\text { Pbest }}_{1,3}$ & Pbest $_{1,4}$ & Pbest $_{1,5}$ \\
\hline & Pbest $_{2,1}$ & Pbest $_{2,2}$ & Pbest $_{2,3}$ & Pbest $_{2,4}$ & Pbest $_{2,5}$ \\
\hline & $\underline{\text { Pbest }}_{3,1}$ & Pbest $_{3,2}$ & Pbest $_{3,3}$ & Pbest $_{3,4}$ & Pbest $_{3,5}$ \\
\hline & $\overline{\text { Pbest }_{4,1}}$ & Pbest $_{4,2}$ & Pbest $_{4,3}$ & Pbest $_{4,4}$ & Pbest $_{4,5}$ \\
\hline & Pbest $_{5,1}$ & Pbest $_{5,2}$ & Pbest $_{5,3}$ & Pbest $_{5,4}$ & Pbest $_{5,5}$ \\
\hline & $\begin{array}{l}\text { Pbest }_{61} \\
\text { Pbest }_{3,1}\end{array}$ & $\begin{array}{l}\text { Pbest }_{6,3} \\
{ }_{1,3} \text { Pbest }_{5,}\end{array}$ & Pbest $_{6,3}$ & Pbest $_{6,4}$ & Pbest $_{6,5}$ \\
\hline
\end{tabular}
randomly generated indices are $\{3,5,1,5,4\}$. Thus, the components of vector Mbest will the components of matrix Pbest whose entries are underlined.

Once Mbest is generated, the corresponding objective function is also evaluated. Also, the objective function corresponding to the worst particle is evaluated. If the mutant particle has the better value of objective function, then the worst particle is replaced by Mbest. It can be expressed as follow:

$$
\text { If } f\left(\text { Mbest }^{k+1}\right)<f\left(\text { Pbest }_{\text {worst }}^{k}\right) \text { then } \quad \text { Pbest }_{\text {worst }}{ }^{k+1}=\text { Mbest }^{k+1}
$$

\subsection{The MPSO- MPPT algorithm for improving the output power of PVs}

The overall flowchart of the proposed MPSO- MPPT method is illustrated in Figure 4.

Firstly, the initial position of particle is chosen by the random value of duty cycle (d) of a boost converter while the fitness function is determined by the output power of PV system. It can be noted that the initial value of (d) should be subjected to upper and lower bounds, [Dmin, Dmax]. Next, a PWM command corresponding to the duty cycle is sent from a digital controller, the output voltage and current are measured, leading to the output power of PV system is calculated. After the fitness of each particle is evaluated, the best initial particle is obtained. Finally, the MPSO algorithm updates the position and velocity of particles based on the personal and overall experience until the optimum value of the duty cycle is obtained. It can be noted that the perturbation of velocity is performed once the tolerance limit has not met. 


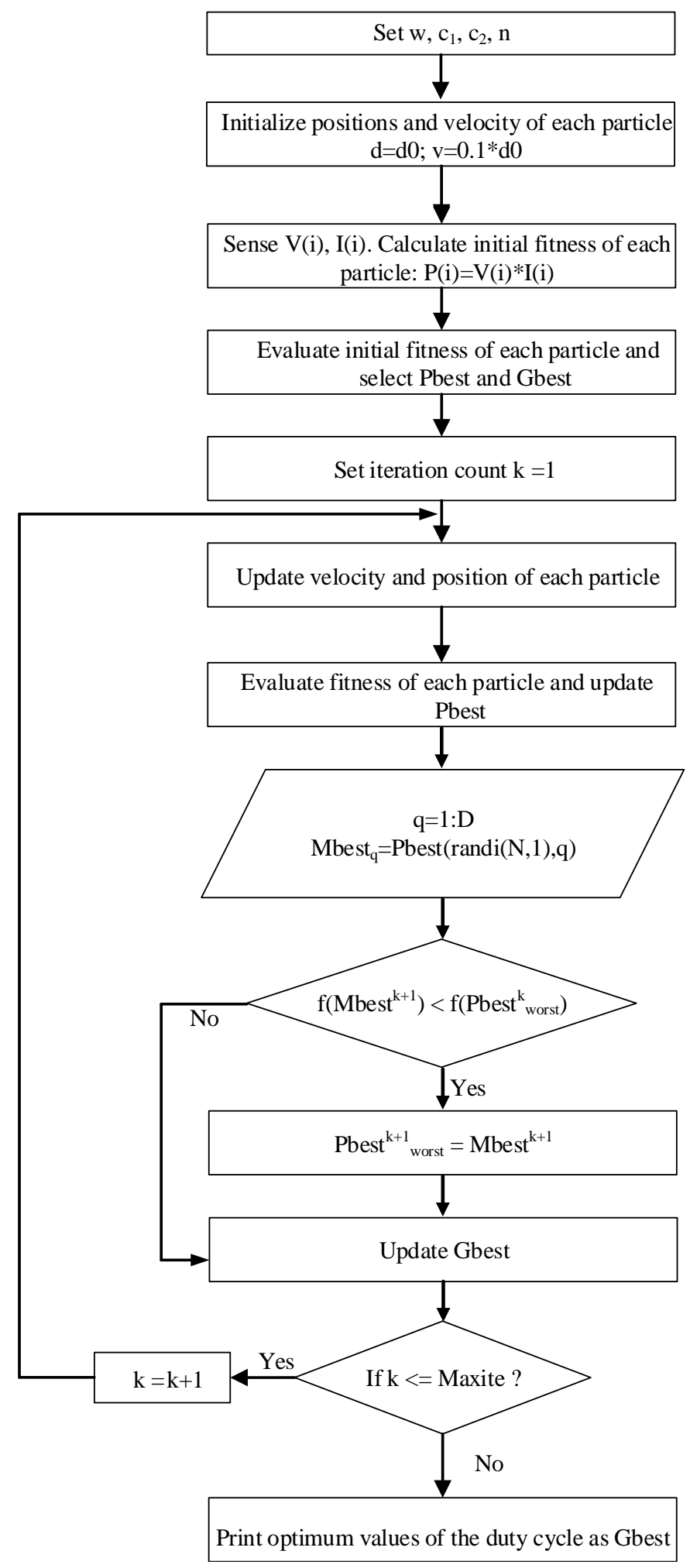

Figure 4. The flowchart of the proposed MPSO- MPPT method

\section{RESULTS AND DISCUSSION}

The proposed MPSO-MPPT algorithm is performed on a model, which consists of a PV solar panel connected to a load through a boost converter with MPPT controller. Figure 5 presents the MATLABSimulink simulation model of the PVs used in this paper. This model is designed and experimented at Control Engineering and Automatic Lab, Ho Chi Minh City University of Transport, as shown in Figure 6: 


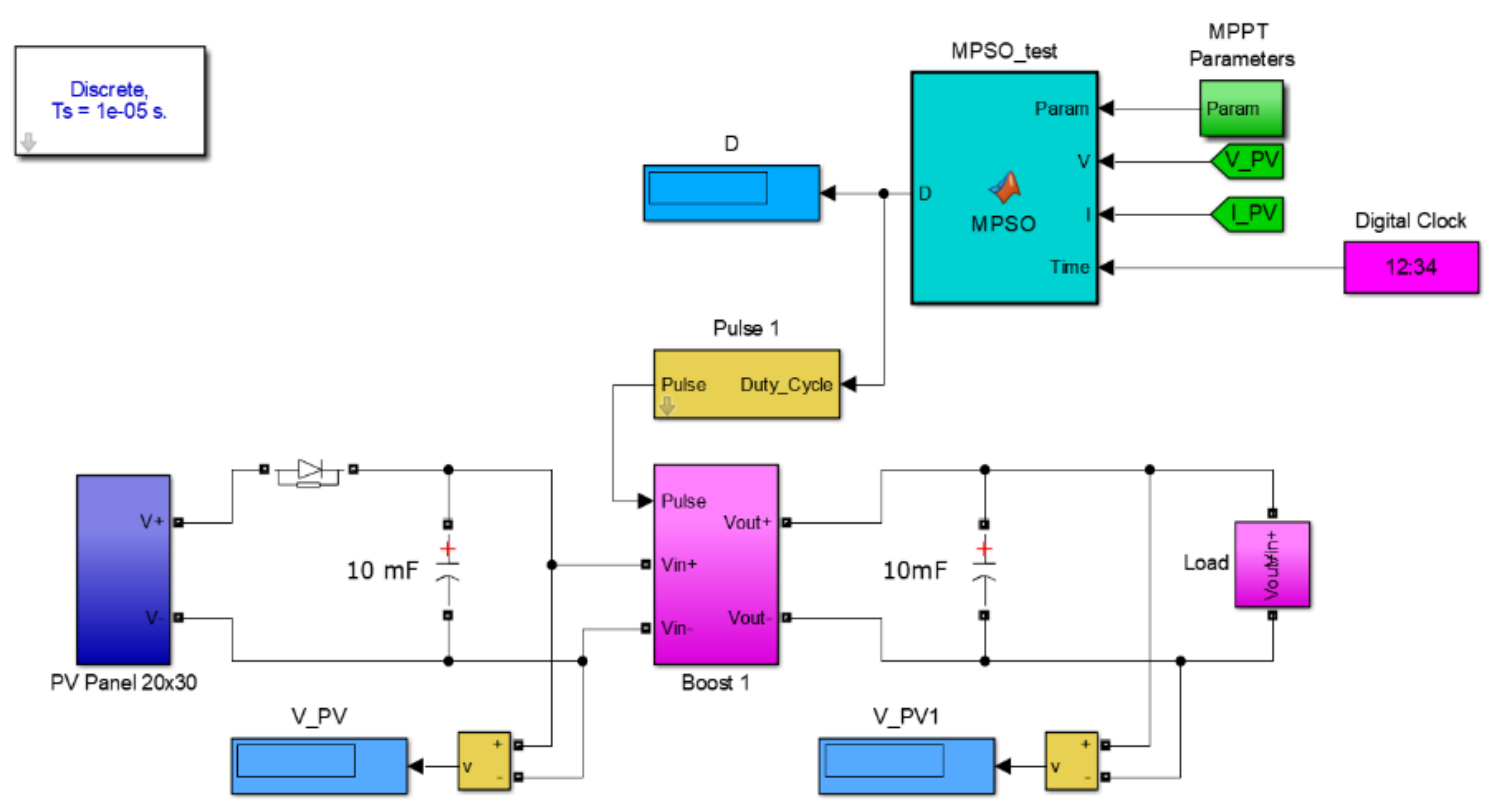

Figure 5. Modelling of the PPSO- MPPT system

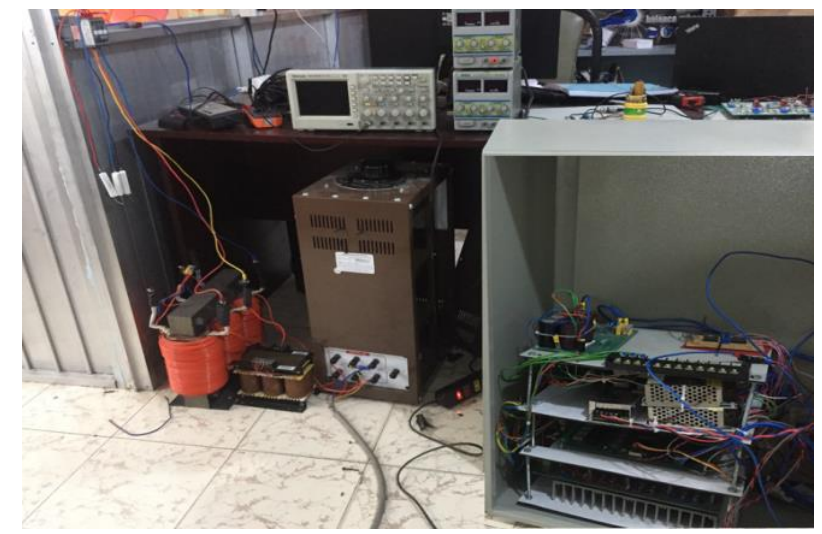

Figure 6. The experimental MPPT system

To implement the proposed MPSO variant, the following parameters have been considered. It is to be noted that these parameters have been selected after performing repeated run by varying them [25].

a) The size of swarm is set as 100

b) The inertia weight belongs the range of 0.4 and 0.9

c) Acceleration factors $\mathrm{c} 1=\mathrm{c} 2=2$

d) Maximum iteration is set to 1000 .

Noted that the programs have been developed in MATLAB environment and executed on an Intel Core i7 processor and $2.66 \mathrm{GHz}$ clock frequency with 8192MB RAM. To demonstrate the effectiveness of the proposed method, the PVs is tested under four different cases, namely 1) without MPPT controller; 2) large fluctuation of insolation; 3) varied load; and 4) PSC. Table 1 compares the maximum power, Pmax generated by the PV array under large step fluctuation of irradiation in case of with and without the MPPT controller.

Table 1. The operating power results without/with the MPPT controller

\begin{tabular}{cccc}
\hline $\mathrm{G}\left(\mathrm{W} / \mathrm{m}^{2}\right)$ & Without MPPT & MPSO-MPPT & The theoretical value of PV \\
\hline 600 & 4567.0 & 5157.5 & 5157.7 \\
700 & 5913.0 & 6009.2 & 6009.7 \\
800 & 6820.0 & 6849.5 & 6850.0 \\
900 & 7360.0 & 7678.0 & 7678.3 \\
\hline
\end{tabular}


It is observed from Table 1 that the operating efficiency of the PV pannel when using the proposed MPSO-MPPT method is greater than $99 \%$ in all test conditions.

Figure 7 shows the dynamic characteristic of the output voltage, current, duty cycle and power during the varied solar insolation at a fixed temperature of $25^{\circ} \mathrm{C}$ after applicating the proposed MPSO. At $\mathrm{t}=$ $2 \mathrm{~s}$, the insolation increases from $400 \mathrm{~W} / \mathrm{m} 2$ to $1000 \mathrm{~W} / \mathrm{m} 2$, the particles (duty cycles) continuously search the new MPP in exploration process, resulting in the large change in output voltage and current. Accordingly, the global power (GP) is tracked accurately at $240 \mathrm{~W}$ after the eighth interation. Similarly, when the solar radiation is decreased from $240 \mathrm{~W} / \mathrm{m} 2$ to $90 \mathrm{~W} / \mathrm{m} 2$ at $\mathrm{t}=6 \mathrm{~s}$, the exploration process starts until it tracks the true MPP at $90 \mathrm{~W}$ after the ninth interation. Moreover, it can be seen from Figure 8 that the MPSO-MPPT algorithm is capability of diminishing the oscillation around the MPP, demonstrates the superiority of the proposed method.

Figure 8 shows the response time of output power in case of the varied load and PSC. The initial level of power is set at $240 \mathrm{~W}$. When the load variation occurs at $t=2 \mathrm{~s}$, there is a significant dip in the output power; however, it can immediately track the true MPP again. At $t=4 \mathrm{~s}$, the PVs is imposed under PSC, the global maximum power is correctly tracked within the seventh interation. In short, the MPSO based MPPT is an efficient method used in case of PSC. It not only removes the the steady-state oscillations around MPP, but also simplier implements with low cost.

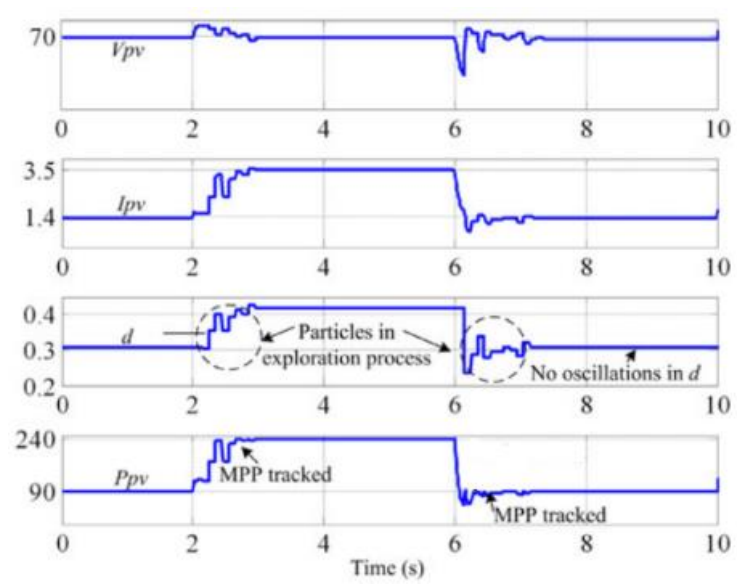

Figure 7. Tracking the MPP under large fluctuation of insolation

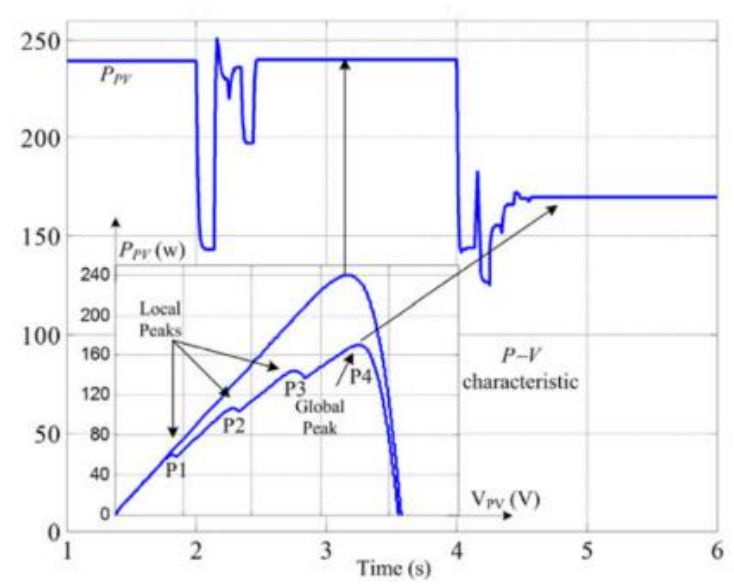

Figure 8. Tracking the MPP under varied load and PSC

Table 2 shows the MPP tracking results using some of diffirent MPPT controllers at a fixed temperature of $25^{\circ} \mathrm{C}$ and the solar insolation of $900 \mathrm{~W} / \mathrm{m} 2$. It can be observed from Table 2 that the maximum operating power using the proposed MPSO algorithm reaches $7678 \mathrm{~W}$, which is better than the obtained results by applying the conventional methods. Furthermore, it takes $0.52 \mathrm{~s}$ for the proposed method to track the MPP, whereas it consumes $0.87 \mathrm{~s}$ for the classical PSO and $2.9 \mathrm{~s}$ for the P\&O and INC, respectively.

Table 2. The MPPT results of using some of difirent MPPT controllers

\begin{tabular}{ccc}
\hline MPPT controllers & Maximum power $(\mathrm{W})$ & Tracking time $(\mathrm{s})$ \\
\hline P\&O & 7655.0 & 2.9 \\
INC & 7656.0 & 2.9 \\
PSO & 7677.2 & 0.87 \\
MPSO & 7678.0 & 0.52 \\
\hline
\end{tabular}

\section{CONCLUSION}

In this paper, a novel MPSO is investigated to improve the operating efficiency of a PVs by replacing the worst particle by a mutant- particle. Overall, the following conclusions can be shown as follows; a) Like the classical PSO, the proposed PPSO-based MPPT is also capability of eliminating the steady-state oscillations to zero value. Furthermore, it can find the MPP accurately under large fluctuations of 
insolation as well as temperature. b) Under load variation and PSC, the proposed MPSO algorithm has the capability of tracking the global optimal peak (the true maximum among the multiple local minimal) that can not be implemented by the conventional methods. c) Tracking the true global peak using the proposed MPSO-based MPPT method is easilier and simpler within a short time in low-cost microcontrollers.

\section{REFERENCES}

[1] N. Muhammad, H. Zainuddin, E. Jaaper, Z. Idrus, An early fault detection approach in grid-connected photovoltaic (GCPV) system. Indonesian J Elec Eng \& Comp Sci, vol. 17, no. 2, pp. 671-679, 2020.

[2] S. L. Brunton, C. W. Rowley, S. R. Kulkarni, and C. Clarkson, Maximum power point tracking for photovoltaic optimization using ripple-based extremum seeking control; IEEE Trans. Power Electron, vol. 25, pp. 2531-2540, 2010.

[3] C. Ali, and A. Chandra, An Optimal Maximum Power Point Tracking Algorithm for PV Systems with Climatic Parameters Estimation; IEEE Trans. Sustain. Energy, vol. 6, pp. 644-652, 2015.

[4] P. Tang and L. Wa, A single cell maximum power point tracking converter without acurrent sensor for high performance vehicle solar arrays; In: Proceeding 36th annual IEEE power electronic specific conference, pp. 165-171, 2005.

[5] K.T. Kobayashi, I. Takano, and Y. Sawada, A study on a two stage maximum power point tracking control of a photovoltaic system under partially shaded insolation conditions; In: Presented at the power engineering society general meeting, 2003.

[6] K. S. Tey and S. Mekhilef, "Modified incremental conductance algorithm for photovoltaic system under partial shading conditions and load variation”, IEEE Trans. Ind. Electron., vol. 61, pp. 5384-5392, 2014.

[7] Elgendy, Mohammed, Z. Bashar, and J. A. David, Operating Characteristics of the P\&O Algorithm at High Perturbation Frequencies for Standalone PV Systems," Energy Conversion, IEEE Transactions, vol. 30, pp.189-198, 2015.

[8] K. Ishaque, Z. Salam, \& G. Lauss, "The performance of perturb and observe and incremental conductance maximum power point tracking method under dynamic weather conditions,“ Applied Energy, vol. 119, pp. 228-236, 2014.

[9] G. Balasubramanian, S. "Singaravelu, Fuzzy logic controller for the maximum power point tracking in photovoltaic system," Int J Comput vol. 41, pp. 22-28, 2012.

[10] Syafaruddin, et al., "Artificial neural network-polar coordinated fuzzy controller based maximum power point tracking control under partially shaded conditions", IET Renew Power Genera, vol. 3, pp. 239-253, 2009.

[11] A. Messai et al., "Maximum power point tracking using GA optimize fuzzy logic controller and its FPGA implementation", Solar Energy, vol. 86, pp. 265-77, 2011.

[12] K. Ishaque, et al., "A novel maximum power point tracking control of photo-voltaic system under partial and rapidly fluctuating shadow conditions using differential evolution", In: Presented at the 2010 IEEE symposium on industrial electronics and applications 2010.

[13] C. K.- Hsiang, L. H. Chien., "Maximum power point tracking method based on modiifed particle swarm optimization for photovoltaic systems", Int J Photoenergy, pp. 1-6, 2013.

[14] K. Ishaque, "A deterministic particle swarm optimization maximum power point tracker for photovoltaic system under partial shading condition”, IEEE Trans Ind Electron, vol. 60, pp. 3195-206, 2013.

[15] K. B. A. Ramdan, Z. F. Ahmed, and E. Adel, "Novel MPPT Algorithm Based on Particle Swarm Optimisation for Photovoltaic Systems", IEEE Transactions on Sustainable Energy, pp. 1949-3029, 2016.

[16] Giraud, F. and Z.M. Salameh, "Analysis of the effects of a passing cloud on a grid-interactive photovoltaic system with battery storage using neural networks", Energy Conversion, IEEE Transactions, vol. 14, pp. 1572-1577, 1999.

[17] Carannante, G., et al., "Experimental Performance of MPPT Algorithm for Photovoltaic Sources Subject to Inhomogeneous Insolation”. Industrial Electronics, IEEE Transactions, vol. 56, pp. 4374-4380, 2009.

[18] Agarwal, H.P.a.V., "MATLAB-Based Modeling to Study the Effects of Partial Shading on PV Array Characteristics". IEEE Transactions on Energy Conversation, vol. 23, pp. 302-310, 2008.

[19] Masafumi Miyatake, M.V., Fuhito Toriumi, Nobuhiko Fujii, Hideyoshi Ko, "Maximum Power Point Tracking of Multiple Photovoltaic Arrays: A PSO Approach.” IEEE trans. On Aerospace and Electro. Sys, vol. 47, 2011.

[20] Phimmasone, V.K., Y., Kamejima, T.,Miyatake, M., "Evaluation of extracted energy from PV with PSO-based MPPT against various types of solar irradiation changes", International Conference on Electrical Machines and Systems (ICEMS), pp. 487-492, 2010.

[21] J. Kennedy, R. C. Eberhart and Y. Shi, Swarm Intelligence, Morgan Kaufmann, San Francisco, USA, 2001.

[22] Ramesh Babu M, C. Venkatesh Kumar, R. Sreekanth, "Optimal volt/var control of distribution system using MOPSO”, Indonesian J Elec Eng \& Comp Sci, vol. 16, no. 3, pp. 1088-1095, 2019.

[23] Thi Thom Hoang, Ming Yuan Cho, Quoc Tuan Vu, "A novel Perturbed Particle Swarm Optimization-based Support Vector Machine for fault diagnosis in power distribution systems", Turkish Journal of Electrical Engineering \& Computer Sciences, vol. 26, pp. 518-529, 2017.

[24] Salam Waley Jeaeb, Abbas Zghair Salman, Qusay A. Jawad, Haider Shareef, “Advanced optimal by PSO-PI for DC motor", Indonesian J Elec Eng \& Comp Sci, vol. 16, no. 1, pp. 165-175, 2019.

[25] Van Huong Dong, Khai Hoan Nhu, Thi Thom Hoang, Thanh Cong Pham, "Tracking maximum power point for photovoltaic system using a novel differential particle swarm optimization”, Journal of Mechanical Engineering Research and Developments, vol. 41, pp. 116-121, 2018. 